\title{
Association of Alpha 1-antitrypsin Deficiency and Genetic Predisposition in Primary Spontaneous Pneumothorax
}

\author{
Taner Sahin ${ }^{1}$, Ibrahim Ethem Ozsoy², Mehmet Akif Tezcan², Derya Kocer ${ }^{3}$ and Murat Erdogan ${ }^{4}$ \\ ${ }^{1}$ Emergency Medicine Clinic, Kayseri City Training and Research Hospital, affiliated with University of Health Sciences, Turkey \\ ${ }^{2}$ Department of Thoracic Surgery, Kayseri City Training and Research Hospital, affiliated with University of Health Sciences, Turkey \\ ${ }^{3}$ Department of Biochemistry, Kayseri City Training and Research Hospital, affiliated with University of Health Sciences, Turkey \\ ${ }^{4}$ Department of Medical Genetics, Kayseri City Training and Research Hospital, affiliated with University of Health Sciences, Turkey
}

\begin{abstract}
Objective: To determine the association of alpha-1 antitrypsin deficiency (AATD) in patients diagnosed with primary spontaneous pneumothorax (PSP), the presence of the SERPINA 1 gene, and the phenotype in patients with low enzyme values.

Study Design: Cross-sectional descriptive study.

Place and Duration of Study: Kayseri City Training and Research Hospital, Turkey, from October 2019 to October 2020.

Methodology: A total of 42 patients with PSP and 42 healthy volunteers were included in the study. The antitrypsin (AAT) level of all participants was measured by the ELISA method. Presence of SERPINA 1 gene was determined in all the participants and its phenotype variants.

Results: In this study, AAT level was statistically and significantly lower in the patient group than the control group ( $p=0.018)$. The presence of the SERPINA 1 gene was studied in 13 (31\%) patients with AATD and 7 (16.7\%) healthy volunteers. Six patients had PI M1V variant (37.5\%), five patients had PI M1A variant (31.3\%), four patients had PI M4 variant (25\%), and one patient had an indeterminate variant (6.2\%). Four healthy volunteers had PI MIV variant (66.7\%), and two healthy volunteers had PI M4 variant (33.3\%).

Conclusion: AAT level was found to be lower in the patient group compared to the control group. In addition, the effect of SERPINA 1 gene on PSP development was found to be benign. AATD is an effective factor in the development of PSP.
\end{abstract}

Key Words: Primary spontaneous pneumothorax, Alpha 1 antitrypsin deficiency, Genotype variants, SERPINA 1 gene.

How to cite this article: Sahin T, Ozsoy IE, Tezcan MA, Kocer D, Erdogan M. Association of Alpha 1-antitrypsin Deficiency and Genetic Predisposition in Primary Spontaneous Pneumothorax. J Coll Physicians Surg Pak 2021; 31(07):775-779.

\section{INTRODUCTION}

Spontaneous pneumothorax (SP), which is the collection of air in the pleural space without trauma, is one of the most common diseases that causes loss of work and power in emergency medicine and thoracic surgery practice. When SP occurs in healthy individuals, it is referred to as primary spontaneous pneumothorax (PSP); and is usually caused by small subpleural blebs bursting in young men. ${ }^{1,2}$ When it occurs in individuals with underlying lung disease [ (chronic obstructive pulmonary disease (COPD), and tuberculosis are the most common causes)], it is defined as secondary spontaneous pneumothorax (SSP); and is seen in the middle-advanced age group. $^{3}$

Correspondence to: Dr. Taner Sahin, Emergency Medicine Clinic, Kayseri City Training and Research Hospital, affiliated with University of Health Sciences, Turkey

E-mail:drmtsahin@gmail.com

Received: April 27, 2021; Revised: May 31, 2021;

Accepted: June 07, 2021

DOI: https://doi.org/10.29271/jcpsp.2021.07.775
Genetically effective factors in the formation of SP have been supported by various evidence. It has been reported that there is a predisposition to SP development in conditions such as alpha-1 antitrypsin deficiency(AATD). ${ }^{4}$ The most common variants of AATD are Z (Glu342Lys) and S (Glu264Val) mutations. The Z mutation causes the most severe plasma deficiency and occurs in more than $95 \%$ of individuals with AATD. ${ }^{5}$ Current evidence suggests that the $\mathrm{Pi} * \mathrm{ZZ}$ genotype is often underrecognised or misdiagnosed by clinicians in individuals with AATD. ${ }^{6}$

Health institutions such as the World Health Organization (WHO), the American and European Thoracic/Respiratory Societies (ATS/ERS) suggest that all COPD patients and adults with irreversible asthma should be tested for AATD at least once in their lifetime. If the alpha- 1 antitrypsin (AAT) serum level is $<110 \mathrm{mg} / \mathrm{dL}$, they recommend genotyping or phenotyping. ${ }^{7}$

In this study, the aim was to determine the AATD in patients diagnosed in the Emergency Medicine Clinic and Thoracic Surgery with PSP; and determine the presence of the SERPINA 1 gene and, if any, the phenotype $(M, Z, S)$ in patients with lowenzyme values.

\section{METHODOLOGY}

The study was initiated after obtaining approval from the 
Erciyes University Faculty of Medicine Ethics Committee with No. 2019/596 and conducted in accordance with the principles of the Declaration of Helsinki. Data on patients and volunteers were prospectively collected over the 12 months between October 2019 and October 2020.

The sample size was determined according to the G*power 3.1.9.7 program (effect size: $0.6, \alpha: 0.05$, power: $0.80, \mathrm{~N} 2 / \mathrm{N} 1: 1$, total sample size: 72 , actual power: 0.80 ). A total of 48 patients, male or female, aged 18-50 were included in the study. Three people with COPD and one person with emphysema, who were considering SSP, two people with acute pneumonia, were excluded from the study. As a result, a total of 42 PSP patients, of either gender aged 18-50 years, who were not previously diagnosed with AATD, were included in the study. In the control group, 42 healthy volunteers, of either gender, aged 18-45 year, who applied to the hospital for routine control, were included. Data of patients and volunteers were recorded in the follow-up form.

For serum AAT level measurement, blood samples were taken from the patients and control groups into gel biochemistry tubes and kept at room temperature for 30 minutes. Blood samples were centrifuged at 3500 rpm for 15 minutes. Serum samples obtained were separated into Eppendorf tubes and stored in a $-80^{\circ} \mathrm{C}$ deep freezer until the study was performed. Serum AAT level was studied in biochemistry laboratory, using enzymelinked immunosorbent assay (ELISA) method, using a commercial kit (Elabscience Biotecnology Inc., TX, USA) according to the manufacturer's instructions. Serum AAT level was expressed as $\mathrm{ng} / \mathrm{mL}$. The kit's measuring range was 3.13-200 ng / mL, with intra / interassay coefficients of variation $<10 \%$. The concentrations of the samples were calculated using the calibration curve obtained from working standards at known levels. 13 patients with AAT level $<110 \mathrm{ng} / \mathrm{mL}$ and seven volunteers were included in the genetic study since the cut-off value for AAT was determined as $110 \mathrm{ng} / \mathrm{mL}$ according to the ROC (Receiver-Operating Characteristic) analysis.

Molecular genetic analysis was performed in medical genetics laboratory to determine the chromosomal phenotype and allele of the SERPINA1 gene from 13 patients and seven volunteers with an AAT value of $<110 \mathrm{ng} / \mathrm{mL}$. For plasma isolation, blood samples were collected in lithium heparin or ethylene diamine tetra acetic acid (EDTA) bottles (Sarstedt Monovette) and centrifuged at $350 \times \mathrm{g} 5 \mathrm{~min}$ at room temperature. Plasma was aliquoted for immediate use or stored at $-80^{\circ} \mathrm{C}$. SERPINA 1 gene sequence method DNA isolation was performed from $2 \mathrm{ml}$ whole blood in the EDTA tube using EZ1 DNA blood $200 \mu$ l kit (Cat. No. 951034- QIAGEN GmbH, Qiagen Straße 1, 40724 Hilden Germany) in EZ1 Advanced XL (Cat. No. 9001492-QIAGEN GmbH, Qiagen Straße 1, 40724 Hilden Germany) device. Primers were designed using the primary blast program (http:// www.ncbi.nlm.nih.gov/tools/primer-blast/, National Center for Biotechnology Information, U.S. National Library of Medicine 8600 Rockville Pike, Bethesda MD, 20894 USA) containing all coding exons (4-7 exons) of the SERPINA 1 (NM_001127701.1) gene. Primers are given in Table I. The exons of the SERPINA 1 gene were reproduced by conducting a conventional PCR with the primed and isolated DNA designed using the Taq PCR master mix kit (Cat. No. 201445- QIAGEN GmbH, Qiagen Straße 1, 40724 Hilden, Germany).

Table I: SERPINA 1 gene primers.

\begin{tabular}{|l|l|l|}
\hline Oligo name & Primer direction & Oligo sequence 5' --> 3' \\
\hline $4 \_1 \mathrm{~F}$ & Forward & TGGCACAGGCTGGTTAAT \\
\hline $4 \_1 \mathrm{R}$ & Reverse & GGAATCTCCGTGAGGTTGAAA \\
\hline $4 \_2 \mathrm{~F}$ & Forward & CCAGTCCAACAGCACCAATA \\
\hline $4 \_2 \mathrm{R}$ & Reverse & ATGGTTGAGAATATTTTCTTG \\
\hline $5 \overline{\mathrm{F}}$ & Forward & CACTCTTCCAAACCTTCACTCA \\
\hline $5 \mathrm{R}$ & Reverse & TCAGTCCCAACATGGCTAAG \\
\hline $6 \mathrm{~F}$ & Forward & TCCCAGAAGAACAAGAGGAATG \\
\hline $6 \mathrm{R}$ & Reverse & AGATACCAGGGTGCAACAAG \\
\hline $7 \mathrm{~F}$ & Forward & CTTACAACGTGTCTCTGCTTCT \\
\hline $7 \mathrm{R}$ & Reverse & GACCAGCTCAACCCTTCTTT \\
\hline
\end{tabular}

General characteristics, disease states, AAT levels, SERPINA 1 gene expression, clinical impact and ages of the patients were expressed as mean, standard deviation, percentage, and frequency, according to the data type. Chi-square analysis was applied to determine whether there is a relationship and dependency between the variables, according to the study groups. In cases where the number of groups is insufficient, probability values for Fisher correction are given. Also, independent samples t-test was used to examine the ATT level according to the groups. ROC analysis was performed to examine the consistency of the cut-off point for diagnosis according to AAT levels. AUROC (Area Under the Receiver Operating Characteristic) values were calculated to compare the ROC fields. Statistical evaluation was made with SPSS (Statistical Package for Social Science, Chicago, II, USA) 19.0 Windows package program, and $\alpha$ $=0.05$ was taken as the critical decision-making value.

\section{RESULTS}

In this study, it was observed that there was no significant difference when the groups were compared according to the ratio of male and female genders. In the study, $11.9 \%$ of the patient group were females and $88.1 \%$ were males. In the control group, $9.5 \%$ were females and $90.5 \%$ were males ( $p>0.999)$. In terms of history, it was observed that $38.1 \%$ had a lung infection in childhood. It was observed that there was a difference though not statistically significant between smoking rates, according to study groups. The rate of smoking in the patient group $(54.8 \%)$ is higher than the control group (40.5\%, $p=0.190)$. The expression rate of SERPINA 1 in the patient groups (31\%) was higher than the control group ( $16.7 \%, p=0.124)$. The benign rates of the clinical effect of SERPINA 1 expression in the patient group $(26.2 \%)$ were higher than the control group $(9.5 \%, p=$ $0.133)$. The study found that the AAT level was statistically significantly lower in the patient group than the control group ( $p$ $=0.018$, Table II).

Alpha-1 antitrypsin deficiency and genetic analysis in primary spontaneous pneumothorax.

In the study, the cut-off point calculated according to the AAT level was found to have a diagnostic accuracy rate of $69 \%$ in patients. According to the AAT level, the cut-off point determined as 110 was found to have a sensitivity value of 0.714 and a specificity value of 0.781 (Table II). 


\begin{tabular}{|c|c|c|c|c|}
\hline \multirow{2}{*}{\multicolumn{2}{|c|}{ Participant characteristics }} & \multicolumn{2}{|l|}{ 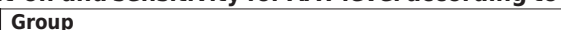 } & \multirow[b]{2}{*}{ p-value } \\
\hline & & \begin{tabular}{|l|} 
Patient group $(n=42)$ \\
$n(\%) /$ mean $\pm S D$ \\
\end{tabular} & \begin{tabular}{|l|} 
Control group $(n=42)$ \\
$n(\%) /$ mean $\pm S D$
\end{tabular} & \\
\hline \multirow{2}{*}{ Gender } & Female & $5(11.9)$ & $4(9.5)$ & \multirow{2}{*}{$>0.999$} \\
\hline & Male & $37(88.1)$ & $38(90.5)$ & \\
\hline \multirow{4}{*}{ Medical history } & Chronic bronchitis & $3(7.1)$ & $0(0)$ & \multirow{4}{*}{$<0.001$} \\
\hline & Asthma & $1(2.4)$ & $0(0)$ & \\
\hline & Lung infection in childhood & $16(38.1)$ & $0(0)$ & \\
\hline & \begin{tabular}{|l|} 
No feature \\
\end{tabular} & $22(52.4)$ & $42(100)$ & \\
\hline Smoking & Yes & $23(54.8)$ & $17(40.5)$ & 0.190 \\
\hline SERPINA1 Expression & Yes & $13(31.0)$ & $7(16.7)$ & 0.124 \\
\hline \multirow{3}{*}{ SERPINA1 clinical ımpact } & Benign & $11(26.2)$ & $4(9.5)$ & \multirow{3}{*}{0.133} \\
\hline & \begin{tabular}{|l|} 
None \\
\end{tabular} & $2(4.8)$ & $3(7.1)$ & \\
\hline & Not Studied & $29(69)$ & $35(83.4)$ & \\
\hline \multicolumn{2}{|l|}{ AAT level * $(\mathrm{ng} / \mathrm{ml})$} & $99.15 \pm 47.00$ & $122.76 \pm 42.40$ & 0.018 \\
\hline \multicolumn{2}{|l|}{ AAT level $($ mean \pm SD) $(\mathrm{ng} / \mathrm{ml})$} & \multicolumn{2}{|l|}{$110.96 \pm 46.05$} & \\
\hline \multicolumn{2}{|l|}{ Age* } & & $32.74 \pm 7.14$ & 0.093 \\
\hline & \multicolumn{2}{|l|}{$\begin{array}{l}29.60 \pm 9.62 \\
31.17 \pm 8.57\end{array}$} & - \\
\hline \multicolumn{2}{|l|}{ Age $($ mean $\pm S D)$} & \multicolumn{2}{|l|}{$0.69[0.56-0.82]$} & \multirow{6}{*}{0.04} \\
\hline \multirow{5}{*}{ AAT Level } & Cut-Off Point(ng/ml) & \multicolumn{2}{|l|}{110} & \\
\hline & & \multicolumn{2}{|l|}{ Sensitivity } & \\
\hline & & \multirow{2}{*}{\multicolumn{2}{|c|}{$\begin{array}{l}0.714 \\
\text { Snerificity }\end{array}$}} & \\
\hline & & & & \\
\hline & & \multicolumn{2}{|l|}{0.781} & \\
\hline
\end{tabular}

Significant difference at 0.05 leve/* Independent samples t-test was applied. AAT: Alpha-1 antitrypsin, SD: Standard deviation, ROC: Receiver-operating characteristic, Cl: Confidence interval.

Table III. Results of SERPINA 1 gene and AAT protein variant analysis in the patient and control group.

\begin{tabular}{|c|c|c|c|c|c|c|c|c|}
\hline Group & $\begin{array}{l}\text { Sequence } \\
\text { number }\end{array}$ & Mutation & Protein name & Exon & Zygosity & rs number & $\begin{array}{l}\text { Clinical } \\
\text { classification }\end{array}$ & \begin{tabular}{|l|} 
Protein \\
variant
\end{tabular} \\
\hline \multirow{16}{*}{ Patient } & \multirow{2}{*}{$\mathrm{P} 1$} & c. $374 \mathrm{G}>\mathrm{A}$ & Arg125His & E4 & Heterozygous & rs709932 & Benign & PI M4 \\
\hline & & C. $1200 A>C$ & Glu400Asp & E7 & Heterozygous & rs1303 & Benign & PI MIV \\
\hline & P2 & c.710T $>C$ & Val237Ala & E5 & Heterozygous & rs6647 & Benign & PI M1A \\
\hline & \multirow{2}{*}{ P4 } & C.710T $>C$ & Val237Ala & E5 & Homozygous & rs6647 & Benign & PI M1A \\
\hline & & c. $1156 C>G$ & Pro386Ala & E7 & Heterozygous & rs12233 & Likely Benign & Unknown \\
\hline & \multirow{2}{*}{ P5 } & c. $374 \mathrm{G}>\mathrm{A}$ & Arg125His & E4 & Heterozygous & rs709932 & Benign & PI M4 \\
\hline & & C. $1200 A>C$ & Glu400Asp & E7 & Homozygous & rs1303 & Benign & PI MIV \\
\hline & \multirow{2}{*}{ P6 } & c. $374 G>A$ & Arg125His & E4 & Heterozygous & rs709932 & Benign & PI M4 \\
\hline & & C. $1200 A>C$ & Glu400Asp & E7 & Homozygous & rs1303 & Benign & PI MIV \\
\hline & P7 & c.710T>C & Val237Ala & E5 & Heterozygous & rs6647 & Benign & PI M1A \\
\hline & P8 & c.710T $>C$ & Val237Ala & E5 & Heterozygous & rs6647 & Benign & PI M1A \\
\hline & P9 & c. $710 \mathrm{~T}>\mathrm{C}$ & Val237Ala & E5 & Heterozygous & rs6647 & Benign & PI M1A \\
\hline & \multirow{2}{*}{ P10 } & c. $374 \mathrm{G}>\mathrm{A}$ & Arg125His & E4 & Heterozygous & rs709932 & Benign & PI M4 \\
\hline & & C. $1200 A>C$ & Glu400Asp & E7 & Heterozygous & rs1303 & Benign & PI M1V \\
\hline & P12 & C. $1200 A>C$ & Glu400Asp & E7 & Heterozygous & rs1303 & Benign & PI MIV \\
\hline & P13 & c. $1200 A>C$ & Glu400Asp & E7 & Heterozygous & rs1303 & Benign & PI M1V \\
\hline \multirow{6}{*}{ Control } & $\mathrm{C} 1$ & C. $1200 A>C$ & Glu400Asp & E7 & Heterozygous & rs1303 & Benign & PI M1V \\
\hline & \multirow{2}{*}{$\mathrm{C} 2$} & c. $374 \mathrm{G}>\mathrm{A}$ & Arg125His & E4 & Homozygous & rs709932 & Benign & PI M4 \\
\hline & & C. $1200 A>C$ & Glu400Asp & E7 & Homozygous & rs1303 & Benign & PI M1V \\
\hline & C4 & C. $1200 A>C$ & Glu400Asp & E7 & Homozygous & rs1303 & Benign & PI M1V \\
\hline & \multirow{2}{*}{$\mathrm{C} 5$} & $c .374 G>A$ & Arg125His & E4 & Heterozygous & rs709932 & Benign & PI M 4 \\
\hline & & C. $1200 A>C$ & Glu400Asp & E7 & Homozygous & rs1303 & Benign & PI M1V \\
\hline
\end{tabular}

In the study, SERPINA 1 gene variant analysis was performed in 13 patients $(31 \%)$ with an AAT value $<110 \mathrm{ng} / \mathrm{ml}$. AAT protein variant was found in $11(26.2 \%)$ of 13 patients. In five patients (11.9\%), it was determined that two different protein variants were present simultaneously. A total of three different AAT protein variants were observed in the patients. It was determined that six patients had PI MIV variant $(37.5 \%)$, five patients had PI M1A variant (31.3\%), four patients had PI M4 variant (25\%), and one patient had SERPINA 1 hg19 variant (6.2\%), and the clinical effect of these variants is benign. ${ }^{8-11}$ SERPINA 1 gene variant analysis was performed in two healthy volunteers (16.7\%) with an AAT value $<110 \mathrm{ng} / \mathrm{ml}$. AAT protein variant was found in 4 $(9.5 \%)$ of seven healthy volunteers. In two healthy volunteers $(4.8 \%)$, it was determined that two different gene variants were present simultaneously. PI M1V variant was found in 4 healthy volunteers $(66.7 \%)$, and PI M4 variant in two healthy volunteers (33.3\%), and the clinical effect of these variants is benign ${ }^{9,10}$ (Table III).

\section{DISCUSSION}

PSP is most common between the ages of 15-34. It is seen as 3.3 to five times more in men than in women. ${ }^{2,12}$ On the other hand, SSP is seen three times higher in men than in women. $^{3}$ There was no difference between the patient and control groups in terms of age and gender in this study. Male gender was higher in both groups. The average age of the patients participating in the study was similar to Dias et al. ${ }^{13}$ In this study, there was a difference though not statistically significant between smoking rates according to study groups. It has been stated in the literature that smoking is the most important environmental risk factor contributing to the development of SP. ${ }^{14}$ Smoking increases the probability of SP development 22-fold in men and 8-fold in women. ${ }^{15}$ In 
this study, it was seen that the rate of SP was 7.4 times higher in men than in women. This rate is higher than some studies in the literature. . $3,12,13$ The authors thought that this may be due to the high rate of cigarette addiction in Turkey and the increase in PSP's tendency because men smoke more, similar to the literature.

In this study, $38.1 \%$ of those with PSP had lung infections in childhood has emerged as an interesting data. There are not enough sources in the literature on this subject, and more studies are needed.

AAT is a prototype of the serine protease inhibitor (SERPIN) protein superfamily and is mainly produced in the liver and reaches the lungs via circulation. ${ }^{16}$ The AAT protein is encoded by the protease inhibitor locus located on chromosome $14 q 32.1$. The most common variants are $S, Z$, and $M$ variants, which are in the form of point mutation. ${ }^{7}$ AATD is an autosomal codominant disease that occurs due to a mutation in the SERPINA 1 gene, and the circulating AAT level is below the protective threshold of $11 \mu \mathrm{mol} / \mathrm{L} .{ }^{17}$ The AAT levels were studied in all individuals participating in this study. The AAT level in the patient group was found to be significantly lower than healthy volunteers. In this study, the presence of the SERPINA1 gene and mutation was also investigated in 13 patients and seven healthy volunteers with AAT level $<110$ $\mathrm{ng} / \mathrm{ml}$, considering AATD. This level is similar to the study of Menga et al. ${ }^{18}$ The proportion of patients suspected of having AATD is higher than that of Dias et al. ${ }^{13}$ This is because PSP was observed in all of the patient groups included in this study and that more PSP patients were included in the current study. Therefore, we think that the low AAT level in this study can be accepted as evidence that it may effectively develop PSP in these 13 patients. On the other hand, AATD can be detected sporadically in entirely healthy individuals, as in this study.

It has been stated in the literature that genetic factors may also be contribute in the development of SP. It has been reported that $10-12 \%$ of patients with SP have familial or sporadic gene mutations. ${ }^{17}$ Ninety percent of people of European descent with normal serum AAT levels have normal M alleles. The genotype of these individuals is called PI MM. More than $95 \%$ of people with severe AAT deficiency carry the homozygous $Z$ allele. Serum AAT levels of those whose genotype is $\mathrm{Pi} Z \mathrm{ZZ}$ are up to $16 \%$ of normal. People whose genotype is ZZ homozygous are at greater risk of emphysema. Serum AAT levels in people with genotype PI SS are $52 \%$ of normal. It has been stated that in individuals whose genotype is PI SZ compound heterozygous, serum AAT levels are $35 \%$ of normal. While the risk of emphysema is absent in PI SS homozygotes, the risk is moderate in Pi SZ heterozygotes. ${ }^{19}$ It was observed that 11 of 13 patients included in this study had an AAT protein variant, five patients had two different gene variants, and one patient had an uncertain protein variant. All of these variants are considered clinically benign. ${ }^{8-11}$ Therefore, it was observed that there was no
SERPINA 1 gene effect in 13 patients with SP who were suspected of having AATD and were genotyped. AAT protein variant was found in $4(9.5 \%)$ of 7 healthy volunteers included in the study. In this study, it was found the SERPINA 1 gene variant rate was higher in the patient group with PSP, similar to the study of Menga et al. ${ }^{18}$ In this study, SERPINA 1 gene variants were heterozygous and had benign clinical effects. These variants are considered clinically benign., ${ }^{9,10}$ Based on these results, it can be concluded that SERPINA 1 protein gene variants can also be seen in completely healthy individuals.

The follow-up of patients with the PI MZ genotype is controversial. The risk of lung disease does not increase in nonsmokers MZ and SZ heterozygotes, but people who smoke and are carriers of PI MM have a significantly increased risk of lung disease than their relatives. ${ }^{19,20}$ A study by Serapinas et al. revealed that $7.7 \%$ of patients with $C P$ had AAT deficiency phenotypes, including the ZZ and SZ phenotypes associated with severe deficiency. ${ }^{17}$ In this study, Z (Glu342Lys) and S (Glu264Val) mutations, which are the most common AATD variants, and whose association with COPD and panacinar emphysema, have been previously determined, were not found in both PSP patients and healthy volunteers.

Since AAT is an acute phase reactant, it can increase during SP formation. Therefore, in this study, if genetic analyses were conducted in some patients with increased AAT levels when PSP occurred, perhaps the presence of the SERPINA 1 gene would have been detected. The authors recommend conducting genetic studies in future studies to investigate the presence of the SERPINA 1 gene in patients with Iow AAT levels after the complete recovery of PSP patients.

\section{CONCLUSION}

The level of AAT was lower in the patient group with PSP. In addition, the effect of the SERPINA 1 gene on PSP formation is benign. Based on these results, AATD may contribute to the development of PSP. Therefore, the authors recommend close follow-up of patients with AATD to prevent or at least reduce the development of possible complications, including COPD, emphysema, and recurrence SP. SERPINA 1 gene variants could be found in patients with PSP and healthy individuals.

\section{ACKNOWLEDGMENTS:}

We want to thank the expert biologist Yasin Ada who contributed to preparing genetic materials in this study. Also, we would like to thank all our healthcare workers who worked devotedly during the COVID-19 pandemic period.

\section{ETHICAL APPROVAL:}

The study was approved by the Faculty of Medicine Ethics Committee, Erciyes University. (Date and No. 2019/596).

\section{PATIENTS' CONSENT:}

Written informed consents were obtained from all participants. 


\section{CONFLICT OF INTEREST:}

The authors declared no conflict of interest.

\section{AUTHORS' CONTRIBUTION:}

TS: Study design, data collection, interpretation of data for the work, discussion, literature review and finalisation of the study. IEO, MAT: Collection, interpretation and analysis of data and discussion.

DK: Biochemical analysis of data and interpretation of results. ME: Genetic analysis of data and interpretation of results.

\section{FUNDING:}

The authors were supported by the Medical Speciality Training Board (TUEK) of our hospital to provide biochemical and genetic kits used in the study.

\section{REFERENCES}

1. Fry WA; Paape K. Pneumothorax. In: Shields TW, LoCicero III J, Ponn RB RV, editor. General Thoracic Surgery. 6th ed. Philadelphia: Lippincott Williams \& Wilkins; 2005. p. 794-805.

2. Hallifax RJ, Goldacre R, Landray MJ, Rahman NM, Goldacre MJ. Trends in the incidence and recurrence of inpatient-treated spontaneous pneumothorax, 1968-2016. Jama 2018; 320(14):1471-80. doi: 10.1001/jama.2018.14299.

3. Onuki T, Ueda S, Yamaoka M, Sekiya Y, Yamada H, Kawakami $\mathrm{N}$, et al. Primary and secondary spontaneous pneumothorax: Prevalence, clinical features, and in-hospital mortality. Can Respir J 2017; 2017:6014967. doi: 10.1155/ 2017/6014967.

4. Scott RM, Henske EP, Raby B, Boone PM, Rusk RA, Marciniak S). Familial pneumothorax: Towards precision medicine. Thorax 2018; 73(3):270-6. doi: 10.1136/thoraxjnl-2017211169.

5. Boone PM, Scott RM, Marciniak SJ, Henske EP, Raby BA. The genetics of pneumothorax. Am J Respir Crit Care Med 2019; 199(11):1344-57. doi: 10.1164/rccm.201807-1212Cl.

6. Mostafavi B, Diaz S, Piitulainen E, Stoel BC, Wollmer P, Tanash HA. Lung function and CT lung densitometry in 37-to 39-year-old individuals with alpha-1-antitrypsin deficiency. Int J Chron Obstruct Pulmon Dis 2018; 13:3689. doi: 10.2147/COPD.S167497.

7. European RS, Society AT. American thoracic society/european respiratory society statement: Standards for the diagnosis and management of individuals with alpha- 1 antitrypsin deficiency. Am J Respir Crit Care Med 2003; 168(7): 818. doi: 10.1164/rccm.168.7.818.

8. National Center for Biotechnology Information. ClinVar; [VCV000017955.3], [Internet]. [cited 2021 May 30]. Available from: http://www.ncbi.nlm.nih.gov/clinvar/variation/ VCV000017955.3

9. National Center for Biotechnology Information. ClinVar;
[VCV000017958.3], [Internet]. [cited 2021 May 30]. Availa ble from : http://www.ncbi.nlm.nih.gov/clinvar/variation/VCV00001795 8.3

10. National Center for Biotechnology Information. ClinVar; [VCV000017957.3], [Internet]. [cited 2021 May 30]. Availa ble

from : http://www.ncbi.nlm.nih.gov/clinvar/variation/VCV00001795 7.3

11. Kopanos C, Tsiolkas V, Kouris A, Chapple CE, Aguilera MA, Richard Meyer MA. VarSome: The Human Genomic Variant Search Engine. [Internet]. Oxford Bioinformatics,. 2018 [cited 2021 Feb 18]. p. bty897. Available from: http://varsome.com/variant/hg19/SERPINA1\%3Ac.1156C\%3 EG

12. Bobbio A, Dechartres A, Bouam S, Damotte D, Rabbat A, Régnard JF, et al. Epidemiology of spontaneous pneumothorax: Gender-related differences. Thorax 2015; 70(7): 653-8. doi: 10.1136/thoraxjnl-2014-206577

13. Dias E, Farinha I, Costa F. Alpha-1-antitrypsin deficiency (AATD) and spontaneous pneumothorax: Guidelines do not recommend screening for AATD in patients with pneumothorax-What did we find in 10 years of clinical evidence? Pulmonol 2021; 27(1):80-1. doi: 10.1016/j.pulmoe.2020. 05.014 .

14. Noppen M. Spontaneous pneumothorax: epidemiology, pathophysiology and cause. Eur Respir Rev an Off J Eur Respir Soc 2010; 19(117):217-9. doi: 10.1183/090591 80.00005310 .

15. Wakai AP. Spontaneous pneumothorax. BMJ Clin Evid 2011; 2011:1505.

16. Lomas DA, Hurst JR, Gooptu B. Update on alpha-1 antitrypsin deficiency: New therapies. J Hepatol 2016; 65(2): 413-24. doi: 10.1016/j.jhep.2016.03.010.

17. Serapinas D, Obrikyte V, Vaicius D, Balciuviene R, Valavicius A, Sakalauskas R. Alpha-1 antitrypsin deficiency and spontaneous pneumothorax: Possible causal relationship. Pneumologia 2014; 63(1):32-5.

18. Menga G, Girbal MS, Montoto Piazza L, Fernández ME. Deficiencia de alfa-1 antitripsina y neumotórax espontáneo ¿solo una coincidencia? [Alpha-1 antitrypsin deficiency and spontaneous pneumothorax. Just a coincidence?]. Medicina (B Aires). 2020; 80(5):473-8. Spanish. PMID: 33048791.

19. Molloy K, Hersh CP, Morris VB, Carroll TP, O'Connor CA, Lasky-Su JA, et al. Clarification of the risk of chronic obstructive pulmonary disease in $\alpha 1$-antitrypsin deficiency PiMZ heterozygotes. Am J Respir Crit Care Med 2014; 189(4): 419-27. doi: 10.1164/rccm.201311-19840C.

20. Ferkingstad E, Oddsson A, Gretarsdottir S, Benonisdottir S, Thorleifsson G, Deaton AM, et al. Genome-wide association meta-analysis yields 20 loci associated with gallstone disease. Nat Commun 2018; 9(1):1-11. doi: 10.1038/ s41467-018-07460-y. 